\title{
Bivariate Analysis of Extreme Wave and Storm Surge Events. Determin- ing the Failure Area of Structures
}

\author{
Panagiota Galiatsatou* and Panagiotis Prinos
}

Department of Civil Engineering, Aristotle University of Thessaloniki, Thessaloniki 54124, Greece

\begin{abstract}
In the present paper a bivariate process of extreme waves and storm surges at a Dutch station on the North Sea is considered. A bivariate logistic model and a sequential estimation procedure are used to extract joint exceedance probabilities of the two variables. The parameters of the margins of the bivariate distribution are defined by three different methods of estimation: a) the Maximum Likelihood Estimation (MLE) approach, b) a Bayesian procedure with flat prior distributions and c) the L-Moments (LM) estimation procedure. Comparison of the results of the three methods is performed and general conclusions are extracted. An approach to estimate the failure area of a particular structure under extreme sea conditions is presented, using the margins resulting from the three different estimation methods.
\end{abstract}

Keywords: Bivariate process, joint exceedance probabilities, Maximum Likelihood Estimation, Bayesian procedure, LMoments, failure area.

\section{INTRODUCTION}

Offshore and coastal structures are exposed to extreme wave conditions and therefore an optimum design requires the estimation of factors affecting them and especially the estimation of extreme oceanographic data. For such systems, the notion of return period has to be extended to more than one dimension to represent multiple environmental variables. The aforementioned structures are often designed based on the estimation of their failure probabilities under extreme conditions of the wave climate. Long-term characteristics of the wave climate (such as the significant wave height or the wave period) and the storm surge can constitute the hydraulic boundary conditions related to the design of offshore and coastal structures.

Structures typically fail because of the occurrence of extreme values of a single environmental process or a critical extreme combination of constituent variables. Failure of a coastal structure is mainly caused by loadings arisen from extreme waves and water levels. The risk of local flood events is related straightforward to nearshore wave and water level conditions. However, the fact that nearshore waves can be affected by hydraulic events, such as the breaking of waves caused by the depth, enforces an extreme value analysis of offshore data. The results of the extrapolation process, deriving from the extreme value analysis of offshore waves and water levels, can then propagate shoreward using wave propagation and long-wave models.

Extreme offshore wave heights are often strongly dependent on high sea levels. The observed water level is the sum of a deterministic astronomical tidal component and a

*Address correspondence to this author at the Department of Civil Engineering, Aristotle University of Thessaloniki, Thessaloniki 54124, Greece; Tel: +30 2310995 856; Fax: +30 2310920 718;

E-mail: pgaliats@civil.auth.gr stochastic meteorologically induced component, the surge. Dependence between surges and waves is expected, since both are related to local weather conditions [1]. Especially at extreme levels strong dependence is likely, when meteorological systems which generate extreme surges also cause strong onshore winds from a direction having a long fetch. For a probabilistic design and optimization of the design process based on flood risk to be possible, loads imposed on coastal and offshore structures are described using a joint density distribution function.

The basic methodology for creating such a multivariate function starts with choosing independent multivariate observations, according to data availability in each particular case and the purpose of such an analysis. If the study variables are primary variables causing the phenomenon of coastal flooding (such as wave height and surge), different possible combinations of concomitant observations have to be studied to find the most conservative among them. To define these possible bivariate observations, thresholds for both primary variables have to be defined. After defining the extreme bivariate observations, dependence between these two variables is calculated. Based on the dependence function of the variables, appropriate bivariate models are chosen to simulate their extreme values. Following the estimation of an appropriate bivariate model, extrapolation to more extreme levels than those observed is conducted. To estimate the marginal parameters of the variables under study, the univariate estimation procedures can be utilized, such as the Maximum Likelihood Estimation, the Bayesian approach and the L-Moments procedure.

To calculate the joint probabilities of wave height and water level or of wave height and surge, different techniques of the Multivariate Extreme Value Theory are utilized. The Multivariate Extreme Value Theory is more complicated than the equivalent univariate analysis and the appropriate methodology to estimate the multivariate models and the 
extrapolation to levels more extreme than those observed, was developed during the last few years. Coles and Tawn [2, 3], Joe et al. [4], Zachary et al. [5], DEFRA [6], Butler [7] and $\mathrm{Li}$ and Song [8] present different aspects of the Multivariate Extreme Value Theory with applications to oceanographic and environmental datasets. Yeh and $\mathrm{Ou}$ [9] use the Multivariate Extreme Value Theory to estimate the joint exceedance probabilities of extreme significant wave height and water level, during hurricanes.

The implementation of the Multivariate Extreme Value Theory is based on the calculation of the dependence between the variables under study. Primitive methods for estimating the dependence of extreme values of different variables have been proposed by Buishand [10, 11], Dales and Reed [12] and Chilès and Delfiner [13]. Coles et al. [14] and Coles [15] use methods of Multivariate Extreme Value Theory to examine the existence of dependence between the bivariate extreme values of significant wave height and surge at a coastal site of southwestern England, while Ledford and Tawn [16] present a range of diagnostic tools based on tail characteristics of joint survivor functions for identifying a structure within extreme events and apply this technique to rainfall and exchange rate data. Svensson and Jones [17] implement the dependence measure $\chi$, in investigating the dependence between the sea surge and river flow, using precipitation as a proxy for river flow.

In the present work a simple methodology to estimate the failure area of adequately defined coastal structures is implemented. This approach selects the "worst case" combination of wave height and surge in terms of the response of the structure under study (e.g. overtopping or force). Another important aspect of the study is that the joint return levels that represent the worst case combination of wave height and surge are calculated using three different methods of estimation of the marginal distributions of both variables, namely the Maximum Likelihood Estimation (MLE) procedure, a Bayesian approach with flat priors and the L-Moments (LM) approach, and the results of these methods are compared. Galiatsatou and Prinos [18] study the bivariate process of extreme wave heights and storm surges, using different methods of selecting concurrent observations as well as different measures of extremal dependence of the two variables involved. Galiatsatou [19] uses the three abovementioned estimation procedures to calculate the parameters of the margins of a bivariate distribution of extreme wave heights and storm surges, considering the cases of full and asymptotic dependence. Galiatsatou and Prinos [20], Galiatsatou et al. [21] and Sánchez - Arcilla et al. [22] consider the effects of different methods of estimation of the marginal distributions of rainfall, surge and wave height data, respectively, on the return levels of the studied process.

The datasets used in the present paper are presented in Section 2, with special reference to the methodology used to "de-cluster" the available bivariate observations. In Section 3 , a methodology to perform bivariate analysis of extreme wave heights and surges is developed. Techniques of selecting bivariate observations, fitting marginal distribution functions to wave heights and storm surges, estimating the dependence of the bivariate data, and selecting and fitting an appropriate model from the family of Multivariate Extreme
Value (MVE) distributions to the bivariate data are presented and analysed further. Three different methods of estimation, the Maximum Likelihood Estimation (MLE) procedure, a Bayesian approach with flat priors and the L-Moments (LM) approach are used to calculate the parameters of the marginal distributions of both variables. At the end of the Section, an approach to determine the failure area of a structure and to estimate the design parameters under extreme sea conditions is presented. The characteristics of the structure used are those proposed by Coles and Tawn [3]. In Section 4, the methodology analyzed in the previous Section is implemented using wave height and storm surge data from a Dutch station on the North Sea. Joint exceedance probabilities resulting from the three different estimation methods of the marginal distributions of wave heights and storm surges (MLE, Bayesian approach, L-Moments) are compared and discussed. The three different estimation methods are also compared with regard to bivariate return levels of wave height and storm surge corresponding to the worst case in terms of overtopping of the studied structure. Finally, Section 5 summarizes the conclusions of the present work.

\section{DATA BASE}

The datasets used in the present work consist of a sequence of 23 years, over the period 1979-2001, from 9 locations along the Dutch coast (Fig. (1)). Two of the nine buoys lie far from the coast in the neighborhood of platforms, while of the other seven, four lie in the deep water some twenty kilometres off the coast, and the rest is in the neighborhood of the coast or the delta estuary. Three hourly data of wave height $\mathrm{H}_{\mathrm{m} 0}$ and its standard deviation, average wave period $\mathrm{T}_{\mathrm{m} 02}$, main wave direction $\mathrm{T}_{\mathrm{h} 0}$, average wave height and period of the highest third part $\mathrm{H}_{1 / 3}$ and $\mathrm{T}_{\mathrm{H} 1 / 3}$ and the wave height of the low frequency waves $\mathrm{H}_{\mathrm{TE} 3}$ are available. In addition to the wave parameters, data on wind speed and direction, water level relative to NAP/MSL, set-up or surge (the difference between the observed and the astronomical water level) and a column indicating the origin of the measured variables are included in the files. At most locations, two wave survey instruments, namely a main sensor and a secondary sensor, were used. If both sensors have registered values for a parameter, then the mean value is given. If, however, data are only present from one sensor, then this is used in the file and if neither of the sensors have values, then estimated values are retained. Missing records are patched by hindcasting. Details of these data sets are given in www.golfklimaat.nl of the Dutch National institute RIKZ. In Fig. (1) the coordinates of the nine stations are presented in the Dutch RD coordinate system (Rijksdriehoeksmeting), while Table 1 contains the names and the geographical coordinates of the stations, the duration of the measurements, as well as the water depth at the nine locations.

The theory described in the following assumes independence of successive observations of wave heights and storm surges. In practice, there is considerable short term dependence in metocean data. Extreme events are typically to be found in storms, which may last for many hours or even several days. In the present paper, an attempt to "de-cluster" extreme wave height and storm surge events similar to the most commonly used approach of explicitly identifying clusters of storm events (a cluster of storm events is a group of 
Table 1. Names, Coordinates and Depth of the Nine Dutch stations and Measurements' Duration

\begin{tabular}{|c|c|c|c|c|c|c|c|}
\hline Full Name of the Station & Name & $\mathbf{x}(\mathbf{k m})$ & $\mathbf{y}(\mathbf{k m})$ & Latitude & Longitude & Duration & Depth (m) \\
\hline K13 platform & K13 & 10.18 & 583.33 & $53^{\circ} 13^{\prime} 04^{\prime \prime}$ & $3^{\circ} 13^{\prime} 13^{\prime \prime}$ & $1979-2001$ & 30 \\
\hline Eierlandse Gat & Eld & 106.51 & 587.99 & $53^{\circ} 16^{\prime} 37^{\prime \prime}$ & $4^{\circ} 39^{\prime} 42^{\prime \prime}$ & $1979-2001$ & 26 \\
\hline IJmuiden munitiestortplaats & Ym6 & 64.78 & 507.67 & $52^{\circ} 33^{\prime} 00^{\prime \prime}$ & $4^{\circ} 03^{\prime} 30 "$ & $1979-2001$ & 21 \\
\hline Euro platform & Eur & 9.96 & 447.6 & $51^{\circ} 59^{\prime} 55^{\prime \prime}$ & $3^{\circ} 16^{\prime} 35^{\prime \prime}$ & $1979-2001$ & 32 \\
\hline Lichteiland Goeree & Leg & 36.78 & 438.79 & $51^{\circ} 55^{\prime} 33^{\prime \prime}$ & $3^{\circ} 40^{\prime} 11^{\prime \prime}$ & $1979-2001$ & 21 \\
\hline Schouwenbank & Swb & 11.24 & 419.52 & $51^{\circ} 44^{\prime} 48^{\prime \prime}$ & $3^{\circ} 18^{\prime} 24^{\prime \prime}$ & $1979-2001$ & 20 \\
\hline Scheur west & Scw & -7.8 & 380.64 & $51^{\circ} 23^{\prime} 32^{\prime \prime}$ & $3^{\circ} 02^{\prime} 57^{\prime \prime}$ & $1979-2001$ & 15 \\
\hline
\end{tabular}

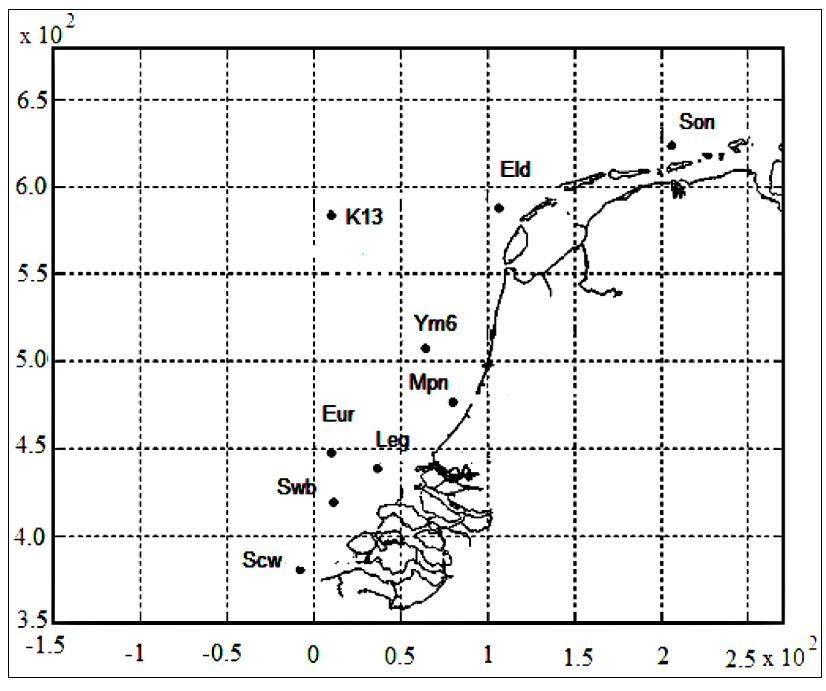

Fig. (1). Field stations at the Dutch coast.

neighbouring excesses of an appropriately defined threshold which begins when the variable under study gets above the threshold and ends when it goes below it) is used. This approach is in fact more easy to apply compared to the fully developed approach of selecting clusters and then choosing the most extreme observation from within each such cluster. The interval over which the data are collected is divided into blocks or periods of equal length. Smith [23] indicated that the results of the analysis were invariant to the values of the time interval $\tau$, apart from the values outside a certain range, which can lead to results of really bad quality. In the literature, time intervals of $24 \mathrm{~h}, 48 \mathrm{~h}, 60 \mathrm{~h}$ or $72 \mathrm{~h}$ are preferred. Within each such period $i$, a multivariate observation $\mathbf{X}_{i}$ is associated, which is defined to be the most extreme of the original (three-hourly) multivariate observations made during that period. Galiatsatou and Prinos [24] analyzing storm surge data from different stations of the Dutch coast suggested that the level of spatial dependence at extreme levels is largely insensitive to the degree of temporal aggregation, for aggregation periods of one day, two days and a week and selected a time period of $24 \mathrm{~h}$ to define independent observa- tions. Taking into account that storm events in the North Sea last at least $24 \mathrm{~h} \mathrm{[25],} \mathrm{the} \mathrm{interval} i$ is identified with a period of one day.

\section{METHODOLOGY}

\subsection{Bivariate Analysis of Extreme wave Heights and Surges \\ 3.1.1. Selection of Bivariate Observations}

In the present study, as discussed above, a time interval of 24 hours is considered to deal with short range dependence in a rather simple way and not to waste a large amount of observations. But in reality, maximum waves and surges do not necessarily occur at the same time and usually there exists a time lag between them. Thus, the selection of daily maximum values of wave heights and storm surges can lead to very conservative results. To overcome this difficulty, four different methods of selecting daily observations are applied. The three of them consider concurrent observations of wave height and surge, while the fourth one considers observations which don't occur simultaneously. From the three methods using concurrent observations, the first uses the maximum wave height in the daily interval $\tau$ with concomitant surge level, the second utilises the maximum surge level in the daily interval $\tau$ with concomitant wave height, while the third uses the joint events of wave height and surge, which give the maximum structural response in the interval $\tau$ of 24 hours. Return levels estimated using the latter methodology should be handled with care, since the response function considered is structure dependent and therefore it is not general. The fourth method of selecting observations considers maximum observations of significant wave height and surge in the time interval of 24 hours. Galiatsatou [19] studied the contour lines of the joint survivor function with return periods of 1, 10, 50 and 100 years for the four cases of selecting observations at station Eld (Eierlandse Gat), $20 \mathrm{~km}$ off the Dutch coast and proved that the results of all cases with concomitant observations do not differ significantly from those of the case where daily maxima waves and surges are used. Thus, the bivariate analysis proceeded with bivariate observations of 24-hourly maxima wave heights and surges. 


\subsubsection{Fitting Marginal Distributions for Wave Height and Surge}

If $X_{1}, X_{2}, \ldots, X_{\mathrm{n}}$ is a series of independent random observations of a random variable $X$ with common distribution function $F(x)$ and $Y_{1}, Y_{2}, \ldots, Y_{\mathrm{k}}\left(Y_{\mathrm{i}}=X_{\mathrm{i}}-u\right)$ are the excesses over a high enough threshold $u$, in some asymptotic sense, the conditional distribution of excesses follows the Generalized Pareto Distribution (GPD):

$G(y)=1-\left(1+\frac{\xi(y-u)}{\sigma}\right)^{-1 / 5}$

where $\sigma$ is the modified scale and $\xi$ is the shape parameter of the GPD distribution. An appropriate threshold $u$ is selected for both variables (wave heights and surges), which defines the level upon which an extreme event is defined. Two different methodologies are used for the selection of $u$ : (a) the mean residual life plot of the excesses of different threshold values and (b) the plots of parameters $\sigma$ and $\xi$ for a variety of possible threshold values. The mean residual life plot consists of the points: $\left\{\left(u, \frac{1}{n_{\mathrm{u}}} \sum_{\mathrm{i}=1}^{\mathrm{n}_{\mathrm{u}}}\left(x_{(\mathrm{i})}-u\right): u<x_{\max }\right\}\right.$ where $x_{(1)}, \ldots, x_{\left(n_{u}\right)}$ consist of the $n_{u}$ observations that exceed $u$ and $x_{\max }$ is the largest of the $X_{i}$ [15]. An appropriate threshold value is the value of $u$ above which the mean residual life plot is approximately linear and estimates of $\sigma$ and $\xi$ are constant with $u$. Due to sampling variability, estimates of these parameters will not be exactly constant, but they should be stable after allowance for their sampling errors.

The choice of the threshold values, $u$, for both the variables of wave height and surge is also based, apart from the methodologies used in the univariate analysis and were previously mentioned, on methodologies used in the bivariate framework. The variables of wave height $\left(X_{1}\right)$ and surge $\left(X_{2}\right)$ are transformed to Fréchet margins, so that each have distribution function $F(z)=\exp (-1 / z)$ for $z>0$, to highlight dependence of the two variables at extreme levels and then the radial and angular components, $R$ and $W$, respectively, are defined as:

$R=\left(X_{1}+X_{2}\right) / n$ and $W_{1}=X_{1} /\left(X_{1}+X_{2}\right)$

where $n$ is the number of bivariate pairs of observations. An extreme bivariate event is defined to be any value for which the radial component $R$ is sufficiently large. To extract such events out of the bivariate sample of wave height and surge data, histograms of the angular component $W_{1}$ are constructed for all bivariate pairs exceeding various choices of the radial component $R\left(r_{\mathrm{o}}\right)$. Then, $r_{\min }$ is taken as the smallest value of $r_{\mathrm{o}}$ above which there is apparent stability in the shape of the histograms [4]. After selecting $r_{\text {min }}$, the marginal thresholds of the variables involved are estimated using equation (3) :

$$
u_{\mathrm{j}}=\Psi_{\mathrm{j}}^{-1}\left(n r_{\min }\right), \mathrm{j}=1,2
$$

where $\Psi_{\mathrm{j}}\left(X_{\mathrm{i}, \mathrm{j}}\right)=-\left\{\log F_{\mathrm{j}}\left(X_{\mathrm{i}, \mathrm{j}}\right)\right\}^{-1} \quad$ for $\quad X_{\mathrm{i}, \mathrm{j}} \leq u_{\mathrm{j}}$. Marginal thresholds, estimated utilizing the methodology based on the bivariate framework (equation (3)), are usually close to the unvariate thresholds or higher than them.
Thus, the assumption made about each marginal component (wave heights and storm surges) is that for high enough threshold $u_{\mathrm{j}}$, the marginal distribution of $X_{\mathrm{j}}-u_{\mathrm{j}}$, for $X_{\mathrm{j}}>u_{\mathrm{j}}$ is the Generalised Pareto Distribution (GPD) $F_{\mathrm{j}}(\mathrm{x})=1-\lambda_{\mathrm{j}}\left\{1+\xi_{\mathrm{j}}\left(x-u_{\mathrm{j}}\right) / \sigma_{\mathrm{j}}\right\}_{+}{ }^{-1 / \zeta_{\mathrm{j}}}, x \geq u_{\mathrm{j}}$, where $\lambda_{\mathrm{j}}=1-F_{\mathrm{j}}\left(u_{\mathrm{j}}\right)$. The scale and shape parameters, $\sigma_{\mathrm{j}}$ and $\xi_{\mathrm{j}}$, respectively, can be calculated using three different methods of estimation: a) the Maximum Likelihood Estimation procedure, b) the Bayesian approach and c) the L-Moments estimation procedure.

\subsubsection{The Maximum Likelihood Estimation Procedure}

The Maximum Likelihood Estimation (MLE) approach is a commonly used estimation procedure. Among others, Coles [15] used this approach to estimate the parameters of extreme value distributions fitted to sea level, rainfall as well as to financial data. The likelihood function gives the relative likelihood of the obtained observations, as a function of the parameters $\boldsymbol{\theta}=(\sigma, \xi): L(\boldsymbol{\theta}, x)=\Pi f\left(x_{\mathrm{i}}, \boldsymbol{\theta}\right)$ and where $L$ (or, for numerical convenience $\log L$ ) is maximized with respect to the parameters $\sigma$ and $\xi$.

Maximization of $L(\boldsymbol{\theta}, x)$ with respect to the set of parameters $\boldsymbol{\theta}$, is numerically straightforward and also has the convenience that various standard large sample theory results are available to enable the numerical calculation of standard errors and confidence intervals. If the available sample sizes are large, there seems little doubt that the Maximum Likelihood estimator is a good choice [26].

\subsubsection{The Bayesian Approach}

In the Bayesian setting, parameters $(\sigma, \xi)$ are treated as random variables and prior distributions on them are intended to represent beliefs about their values, prior to the availability of the data. The specification of information in the form of a prior distribution is regarded alternately as the greatest strength and the main pitfall of Bayesian inference [15]. The absence of genuine prior information leads to the use of priors $\pi(\boldsymbol{\theta})$ that have very high variance, or equivalently, are nearly flat. A trivariate normal distribution is used here, that enables the specification of independent parameters. Setting $\varphi=\log \sigma$, a prior density function $f(\sigma, \xi)=f_{\varphi}(\varphi)$ $f_{\bar{\xi}}(\xi)$ is chosen, where $f_{\varphi}(\cdot), f_{\xi}(\cdot)$ are normal density functions with mean zero and very high variances, corresponding to a specification of prior independence in the parameters $\varphi$ and $\xi$. The posterior density of $\boldsymbol{\theta}$ is given by [27]:

$$
\pi(\theta \mid x)=\frac{\pi(\theta) L(\theta ; x)}{\int_{\theta} \pi(\theta) L(\theta ; x) \mathrm{d} \theta} \propto \pi(\theta) L(\theta ; x) \ldots \ldots \ldots \ldots .
$$

where $L(\theta ; x)$ is the likelihood function. Standard Markov chain Monte Carlo methods routinely allow the approximation of integrals such as the one in the denominator of equation (4). More details about the Bayesian methodology used here are given in Galiatsatou and Prinos [20], Galiatsatou et al. [21] and Sánchez-Arcilla et al. [22].

\subsubsection{The L-Moments Approach}

L-Moments are analogous to ordinary moments. They provide measures of location, dispersion, skewness, kurtosis and other aspects of the shape of probability distributions or data samples, but are computed from linear combinations of 
the ordered data values [26]. They were introduced by Hosking [28], who assessed that L-moments weigh each element of a sample according to its relative importance.

The main advantage of L-Moments is that, being a linear combination of data, they are less influenced by outliers and the bias of their small sample estimates remains fairly small. It is therefore anticipated that L-Moments can provide reliable estimates of tail-index with a relatively small sample of the POT data. Furthermore, the required computation is quite limited compared with other traditional techniques, such as the Maximum Likelihood Estimation approach [29].

Using three sample L-Moments, the scale $(\sigma)$ and shape ( $\xi$ ) parameters of the GPD can be estimated as [29]:

$\xi=\frac{\left(3 \tau_{3}-1\right)}{\left(\tau_{3}+1\right)}$

$\sigma=(1-\xi)(2-\xi) \lambda_{2}$

where $\tau_{3}$ is the normalized L-Moment $\tau_{3}=\lambda_{3} / \lambda_{2}$. The LMoments approach is a method requiring quite limited computation compared with other traditional techniques, such as the Maximum Likelihood Estimation approach.

\subsubsection{Estimation of Dependence of the Bivariate Data}

Wave heights and storm surges are not independent variables, but they are certainly characterized by some form and some degree of dependence. The complete pair of measures of extremal dependence $\chi$ and $\bar{\chi}$, introduced by Coles et al. [14], is informative for both asymptotically independent and dependent variables. After transformation of the pair of variables $\left(X_{1}, X_{2}\right)$ to $(U, V)=\left\{F_{X_{1}}\left(X_{1}\right), F_{X_{2}}\left(X_{2}\right)\right\}$ having Uniform marginal distributions, the one-dimensional function $\chi(u)$ is defined for a given threshold as:

$\chi(u)=2-\frac{\log \operatorname{Pr}(U<u, V<u)}{\log \operatorname{Pr}(U<u)}$ for $0 \leq u \leq 1 \ldots \ldots \ldots \ldots \ldots$

The measure $\chi$ can then be defined as:

$\chi=\lim _{u \rightarrow 1} \chi(u)$

The one dimensional function $\bar{\chi}(u)$ is defined for $0 \leq u \leq 1$ :

$\bar{\chi}(u)=\frac{2 \log \operatorname{Pr}(U>u)}{\log \operatorname{Pr}(U>u, V>u)}-1:$

It follows that:

$\bar{\chi}=\lim _{u \rightarrow 1} \bar{\chi}(u)$

When used for bivariate random samples with identical marginal distributions, both measures provide an estimate of the probability of one variable (e.g. wave heights) being extreme, provided that the other one (e.g. surge levels) is extreme. The sign of $\chi(u)$ determines whether the variables are positively or negatively associated at the quantile level $u$. In the special case $\chi=0$ the variables are asymptotically independent. For asymptotically dependent variables $\bar{\chi}=1$. The complete pair of $(\chi, \bar{\chi})$ can give an impression of extremal dependence. The pair $(\chi>0, \bar{\chi}=1)$ indicates asymptotic dependence, while the value of $\chi$ determines the strength of dependence and the pair $(\chi=0, \bar{\chi}<1)$ signifies asymptotic independence, in which case the value of $\bar{\chi}$ determines the strength of dependence within the class [15]. Galiatsatou and Prinos [18] present plots of the pair $(\chi, \bar{\chi})$ for wave heights and surges at station Eld (Eierlandse Gat), $20 \mathrm{~km}$ off the Dutch coast, identifying an increased degree of correlation of the two processes as $u \rightarrow 1$ suggesting a tendency for the most extreme levels to be correlated.

Another measure used in the present paper to examine dependence of the two processes at extreme levels is the coefficient of tail dependence, $\eta$. For estimating the coefficient of tail dependence, $\eta$, the structure variable $T=\min (X, Y)$, where $X$ and $Y$ are the wave height and surge data transformed to have the standard Fréchet distribution, is defined. The coefficient of tail dependence, $\eta$, is estimated by using a point process approach, as the shape parameter of the univariate $T$ variable. The coefficient of tail dependence, $\eta$, can be related to the dependence measure $\bar{\chi}$ using the formula: $\bar{\chi}=2 \cdot \eta-1$. If $\eta=1$ and $L(z) \rightarrow \mathrm{c}$ as $\left.\mathrm{z} \rightarrow \infty(L)^{\prime}\right)$ is a slowly varying function, i.e. $\lim _{s \rightarrow \infty}\{L(s t) / L(s)\}=1$ for all fixed $t>0)$, with $0<c \leq 1$, then $(\chi=c, \bar{\chi}=1)$ and the variables are asymptotically dependent of degree $\mathrm{c}$ [14].

\subsubsection{Choosing an appropriate model from the family of MVE}

To determine an appropriate bivariate extreme value model (BVE), components $X_{1}$ (wave height) and $X_{2}$ (surge), having unit Fréchet margins, are transformed to radial and angular components, $R=\left(X_{1}+X_{2}\right) / n$ and $W_{1}=X_{1} /\left(X_{1}+X_{2}\right)$, where $n$ is the number of bivariate pairs. The $W_{1}$ versus $\log R$ plot, for points exceeding at least one of the marginal thresholds defined can be used to determine whether there is mass near the boundaries of the one dimensional unit simplex $S_{2}$ ( $\left.\left\{S_{2}=\left(W_{1}, W_{2}\right): \sum_{j=1}^{2} W_{j}=1, W_{j}>0, j=1,2\right\}\right)$. If most of the points lie in the interval $0.1<W_{1}<0.9$ and few points are near the boundaries of $W_{1}$, parametric models with all mass in the interior of the one dimensional unit simplex $S_{2}$ are quite appropriate, namely the Dirichlet and symmetric versions of the two logistic models (logistic and negative logistic) [2].

A more detailed inspection of the structure of the dependence function of the bivariate data requires the estimation of the parameter of asymmetry $\psi_{\alpha}$ for all candidate bivariate models. If $\psi_{\alpha}$ is the asymmetry parameter of a bivariate extreme value model, it is estimated using the formula [24]:

$\psi_{\alpha}=\int_{0}^{0} 4\left(A\left(W_{1}\right)-A\left(1-W_{1}\right)\right) /\left(3-2 \sqrt{2)} \mathrm{d} W_{1}\right.$

This measure lies in the interval $[-1,1]$, with large absolute values representing stronger asymmetry. $A$ is the dependence function of the data. For the logistic and negative logistic models $A\left(W_{1}\right)=A\left(1-W_{1}\right)$ for all $0 \leq W_{1} \leq 0.5$, so the value of $\psi_{\alpha}$ will be zero. 


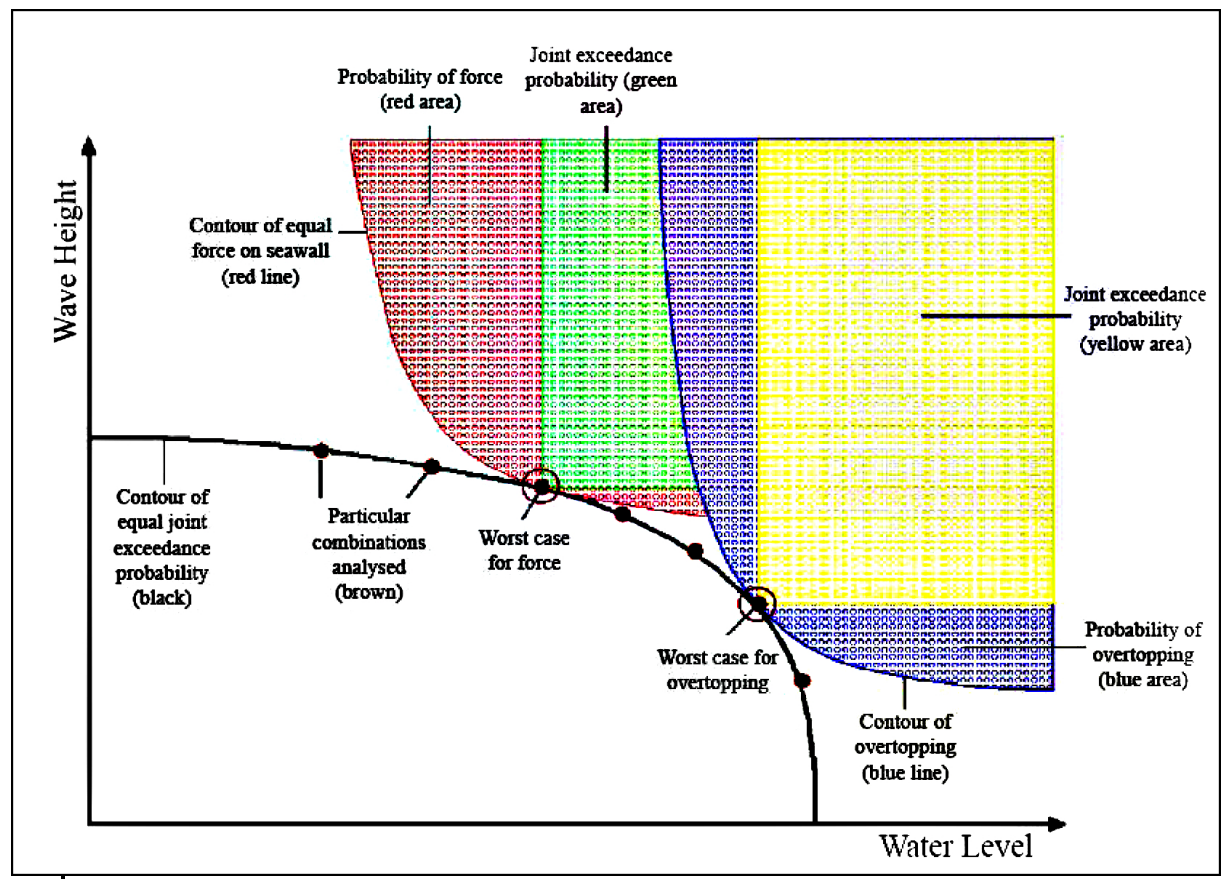

Fig. (2). The relationship between joint exceedance and response probabilities (DEFRA [31]).

An informal impression of the best fitting model can be given by comparing the estimations of the negative loglikelihoods (NLLH), arising from the maximization of the censored likelihood. But testing between different families of dependence models cannot be based formally on likelihood values since the models are not nested [3].

\subsubsection{Fitting the Appropriate Bivariate Model}

Modeling approaches for multivariate extremes are analogous to block maximum, threshold and point process results, derived for univariate extremes. It should be noted that approaches based on threshold exceedances are potentially more efficient than those based on component-wise maxima. The former avoid the wastefulness of data implied in a componentwise block maxima analysis. However, they cannot easily be applied to spatio-temporal data, because they cannot easily be adapted to deal with short-range dependence [7].

The simplest Bivariate Extreme Value distribution is the bivariate logistic model. The bivariate logistic distribution function for wave heights and surges with dependence parameter $r(0<r \leq 1)$ is [30]:

$G\left(x_{1}, x_{2}\right)=\exp \left[-\left(z_{1}^{1 / r}+z_{2}^{1 / r}\right)^{r}\right]$

where $z_{1}$ and $z_{2}$ are the transformed GPD margins of the variables $X_{1}$ (waves) and $X_{2}$ (surges). Complete dependence is achieved in the limit as $r$ approaches zero. Independence is obtained when $r=1$. One of the main properties of the model is the exchangeability of the variables involved. Marginal parameters of the two variables (wave heights and surges) are estimated using the three different estimation procedures analyzed: a) the Maximum Likelihood Estimation procedure, b) the Bayesian approach and c) the L-Moments estimation method. The dependence parameter, $r$, of the bivariate logistic model (11) is estimated using a sequential estimation pro- cedure, after defining the marginal parameters of the distributions of wave heights and surges.

\subsection{Determination of the Failure Area of a Structure - Design Under Extreme Conditions}

If $X_{1}, \ldots, X_{d}$ are random variables corresponding to constituent processes affecting a particular structure with a vector of design parameters $\mathbf{v}$, structural failure can occur if $\mathbf{X}=$ $\left(X_{1}, \ldots, X_{d}\right) \in A_{\mathbf{v}} \subset \mathbf{R}^{d}$, for a failure region $A_{\mathbf{v}}=\left\{\mathbf{x} \in \mathbf{R}^{d}: b(\mathbf{x}\right.$; v) $>0\}$, for a boundary function $b: \mathbf{R}^{d} \times V \rightarrow \mathbf{R}$, where $V$ is the design parameter space of $\mathbf{v}$ [3]. The main problem which arises is, for a given parameter vector $\mathbf{v}$, to determine the probability of failure $p=\operatorname{Pr}\left\{\mathbf{X} \in A_{v}\right\}$, or to define the value of $\mathbf{v}$ which is linked to the probability $p$. This latter problem has unique solutions only if $V \subset \mathbf{R}$. However, the determination of the form of the boundary function $b$ is indeed very difficult and depends on a large variety of factors, like the design structure characteristics under study and the features of the processes affecting it.

Joint exceedence probability refers to the chance of two or more partially related variables occurring simultaneously. Response probability refers to the occurrence of a particular response, like overtopping or failure, which depends on the joint occurrence of those variables. The main discrepancy between joint exceedence and response probabilities lies on the fact that a number of combinations of the variables involved can be derived with a given joint exceedence return period, but only one of these will be the worst case in terms of response and it may not be the same for each response.

In DEFRA [31] there is an example that shows the relationship between joint exceedence probabilities and response probabilities of a coastal structure (Fig. (2)). A number of combinations of waves and sea levels can be derived with a given joint return period (black curve). Only one of these will be the worst case in terms of response. Two different responses are considered in this particular example, overtop- 

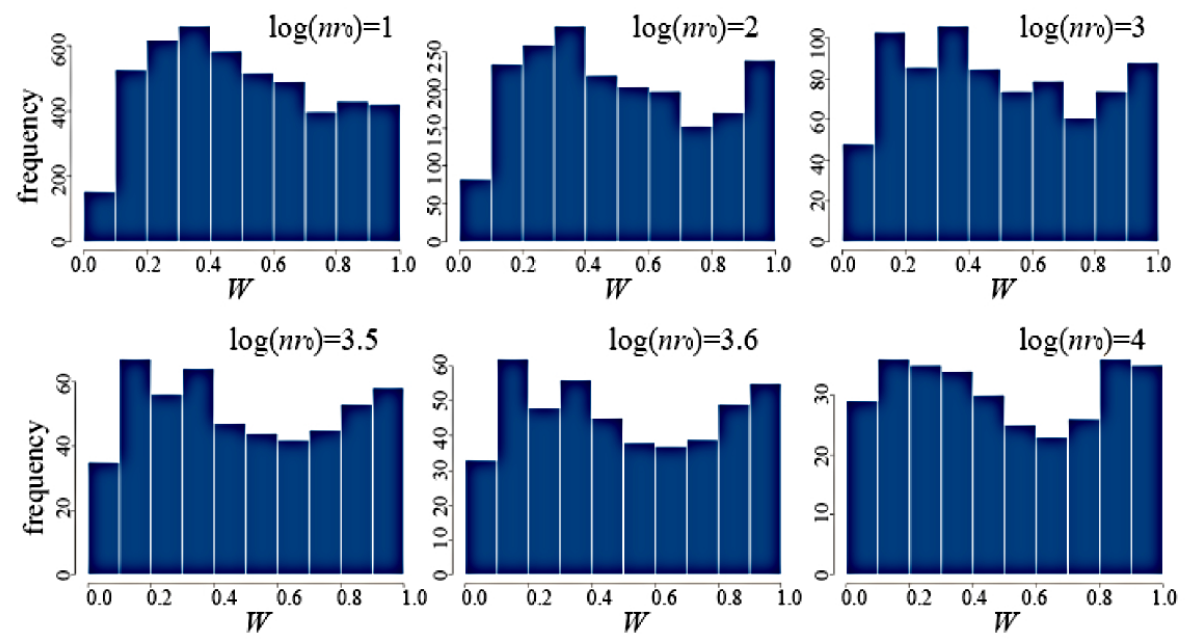

Fig. (3). Histograms of the angular component $W_{1}$ for all exceedances of a radial threshold $r_{0}$, for the bivariate pair of wave heights and surges. The histograms are created for values of $r_{\mathrm{o}}$ : a) $\left.\left.\left.\left.r_{\mathrm{o}}=n^{-1} \exp (1), \mathrm{b}\right) r_{\mathrm{o}}=n^{-1} \exp (2), \mathrm{c}\right) r_{\mathrm{o}}=n^{-1} \exp (3), \mathrm{d}\right) r_{\mathrm{o}}=n^{-1} \exp (3.5), \mathrm{e}\right) r_{\mathrm{o}}=n^{-1}$ $\exp (3.6)$ and f) $r_{\mathrm{o}}=n^{-1} \exp (4)$.

ping (blue curve) and force (red curve). The worst case combination for the structure under study is defined graphically as the intersection of the contour of joint exceedance probability corresponding to a particular probability of exceedance and the contour of equal response to appropriately chosen loadings. The green and yellow areas illustrate ranges of wave height and sea level with the given exceedance probability. The equal overtopping curve lies towards the bottom right of the diagram, where sea level is higher, while the equal force curve lies towards the top left of the diagram, where wave height is higher.

In the present study, the structure utilised is a seawall. Its design characteristics are those presented by Coles and Tawn [3]. For this particular structure, overtopping is the critical cause of flooding of coastal areas behind it. The combination of large wave heights and high surges can contribute to high overtopping rates and thus create extended flooding to the protected areas. Wave heights in the area of the seawall and surges can contribute to the creation of a natural structure variable for the problem under study. Following Coles and Tawn [3], if $X_{1}^{*}, X_{2}{ }^{*}$ are the inshore significant wave height and wave period, respectively, $X_{3}$ is the surge, $v$ is the height of the seawall, $z$ is the mean tidal level and $m$ is the mean sea level at the area, the expected overtopping discharge rate per unit length of the seawall $Q(v, z)$ is:

$$
Q(v, z)=\alpha_{1} g X^{*}{ }_{1} X_{2}^{*} \exp \left\{-\alpha_{2}\left(v-X_{3}-z-m\right) / X_{2}^{*}\left(g X_{1}^{*}\right)^{1 / 2}\right\}
$$

where $g$ is the acceleration of gravity and $\alpha_{1}, \alpha_{2}$ are dimensionless constants depending on the form and characteristics of the seawall design, taking values $\alpha_{1}=0.025$ and $\alpha_{2}=81.5$, as the seawall is considered a rough sloping embankment with gradient 1:5.

The breaking of waves and other phenomena occurring in shallow waters, cause the characteristics of the waves nearshore to vary from those offshore. For this reason, the relationships proposed by Tucker et al. [32] are used to transform to characteristics of the waves offshore, namely the wave height and the wave period:

$$
X_{1}^{*}=X_{1}\left[1-\exp \left\{\frac{-\left(m+z+X_{3}\right)^{2}}{2 X_{1}^{2}}\right\}\right]^{1 / 2} \text { and } X^{*}{ }_{2}=X_{2} \ldots \ldots \ldots
$$

If the design overtopping discharge per unit length is $0.002 \mathrm{~m}^{3} / \mathrm{sm}$ [3], the corresponding boundary function $b(\mathbf{x}$; v) is defined as:

$b(\mathbf{x} ; \mathbf{v})=Q(v, z)-0.002$.

\section{RESULTS}

In the following, the methodology presented in the previous Section, will be implemented to wave height and surge data of station Swb (Schouwenbank), located in the southern part of the Dutch coast (see Fig. (1)). The geographical coordinates of station Swb are 51 $44^{\prime} 48^{\prime \prime}$ (latitude) and $3^{\circ} 18^{\prime} 24^{\prime \prime}$ (longitude) and the water depth is approximately $20 \mathrm{~m}$. Station Swb lies within the greater area of a coastal structure. The methodology was also implemented to other Dutch stations, but due to the fact that the main interest of the study is the presentation of a general methodological framework, the results are not included for the sake of brevity.

Following Galiatsatou [19], four different methods of selecting daily observations of wave heights and storm surges are applied. The three of them consider concurrent observations of wave height and surge, as described in Section 3, while the fourth one considers daily maximum values of the two variables, which do not occur simultaneously. Results of joint return levels and joint exceedance probabilities for all cases with concomitant observations do not differ significantly from those of the case where daily maxima waves and surges are used. Thus, the bivariate analysis at station Swb will proceed with bivariate observations of 24-hourly maxima wave heights and surges.

Univariate thresholds for wave heights and storm surges, using the mean residual life plot and the plots of parameters $\sigma$ and $\xi$ for a variety of possible threshold values, are estimated close to the $95 \%$ quantiles of both variables involved. The bivariate threshold $r_{\mathrm{o}}$ is estimated using the methodology presented in Section 3. Fig. (3) shows histograms of values 
of $W$ with $\log \left(n r_{\mathrm{o}}\right)$ for values of the bivariate threshold $r_{\mathrm{o}}$ equal to $\exp (1) / n, \exp (2) / n, \exp (3) / n, \exp (3.5) / n, \exp (3.6) / n$ and $\exp (4) / n$ at station Swb. Values of $r_{\mathrm{o}}$ which lay between the ones presented in Fig. (3), although estimated, are not presented in the paper. The value of $r_{\mathrm{o}}$ chosen for the analysis is fixed at $r_{\mathrm{o}}=\exp (3) / n$. Such a threshold corresponds, by using equation (3) to marginal thresholds close to the $95 \%$ quantile of wave height and surge elevations at station Swb. It can be noticed that in this particular case, univariate and bivariate thresholds are estimated really close to each other.

To estimate extremal dependence of wave heights and storm surges, the pair of measures $\chi$ and $\bar{\chi}$ introduced by Coles et al. [14], is defined (see Section 3). Fig. (4) shows plots of the functions $\chi(u)$ and $\bar{\chi}(\mathrm{u})$ for station Swb. Mean values as well as $95 \%$ confidence intervals for both functions are included.
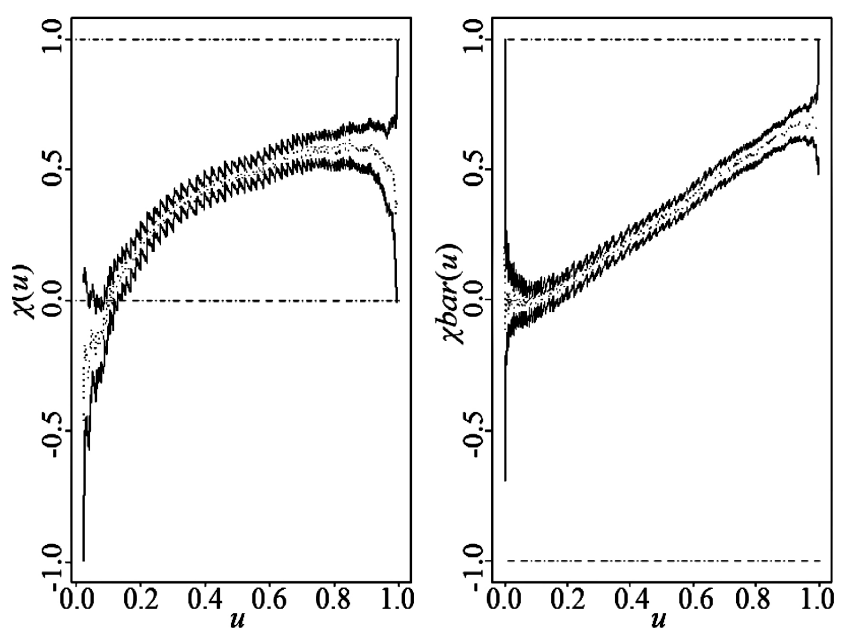

Fig. (4). Mean values and $95 \%$ confidence intervals of functions $\chi(u)$ and $\bar{\chi}(\mathrm{u})$ for the bivariate observations of 24-hourly maximum wave height and surge variables.

It can be concluded that for the greatest range of values of $u, \chi>0$ (Fig. (4)). The $\chi(u)$ function takes negative values only for a small range at the beginning of the range of values of $u$, showing some slight evidence of negative dependence. However, this area is limited. From Fig. (4), considering the $95 \%$ confidence interval, the value $\bar{\chi}=1$ is a possible limit.

Consequently, there is some evidence that wave heights and surges are consistent with asymptotic dependence at extreme levels.

To examine dependence of the two processes at extreme levels, the coefficient of tail dependence, $\eta$ is also estimated (see Section 3). Considering a threshold of $75 \%$ for the $T$ variable, the coefficient of tail dependence, $\eta$, is equal to $\hat{\eta}=0.827$ (standard error $=0.087$ ). Considering a threshold of $95 \%$ of the $T$ variable, the respective value of the coefficient of tail dependence is $\hat{\eta}=0.818$ (standard error = 0.088 ). Thus, if the $95 \%$ confidence intervals for the coefficient of tail dependence, $\eta$, are defined $\hat{\eta} \approx 1$ and therefore the bivariate data of wave height and storm surge can be consistent with asymptotic dependence.
Estimating both the pair of measures $(\chi, \bar{\chi})$ and the coefficient of tail dependence, $\eta$, wave height and surge seem to be consistent with asymptotic dependence. Consequently, multivariate extreme value models from the family of MVE can be used to model the dependence function of the variables and to estimate their joint distribution function. To determine an appropriate bivariate extreme value model (BVE) for wave height and storm surge data, the $W_{1}$ versus $\log R$ plot of bivariate points exceeding at least one threshold, the asymmetry parameter, $\psi_{\alpha}$, and the estimates of the likelihood function for all candidate models are used as evidence. Fig. (5) shows a $W_{1}$ versus $\log R$ plot of bivariate points exceeding at least one threshold for the pair of wave height and storm surge data at station Swb. It can be concluded that few points lay near the boundaries of $W_{1}$, suggesting a need to use a bivariate model with all mass in the interior of the simplex $S_{2}$. Estimates of $\psi_{\alpha}$ do not show evidence of significant asymmetry in the dependence structure of the data. Considering the previous finding, together with the estimates of the likelihood function for all candidate models (Dirichlet, symmetric logistic and symmetric negative logistic model), the symmetric logistic model is suggested to be fitted to the bivariate data.

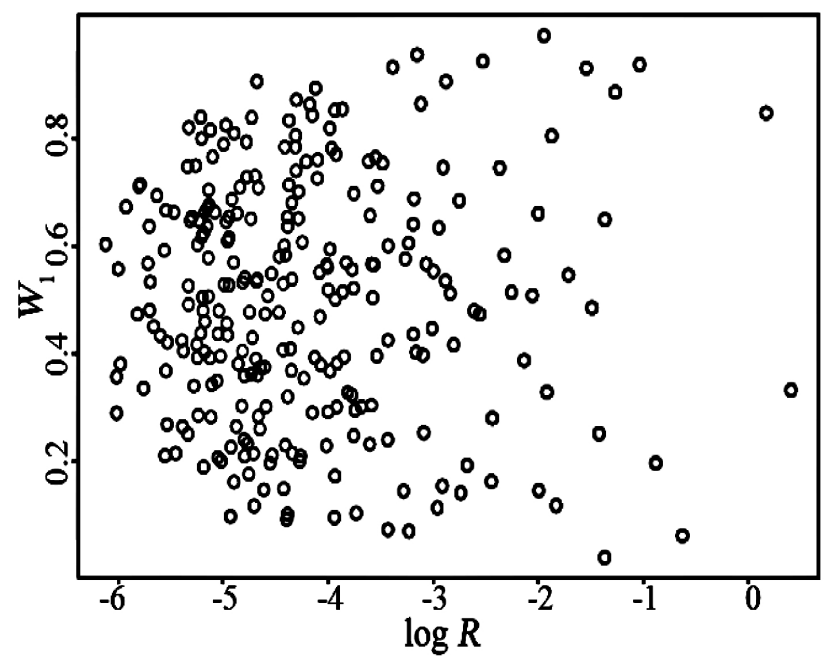

Fig. (5). $W_{1}$ versus $\log R$ plot for points exceeding at least one threshold.

Fig. (6) shows contour lines of bivariate return levels for return periods of $1,10,50,100$ and 10000 years with Maximum Likelihood, Bayesian, and L-Moments estimators of the parameters of the extreme value models.

Table 2 presents the maximum values of wave height and surge return levels at station Swb for return periods of 1, 10, 50, 100 and 10000 years, with GPD margins for the two variables involved, estimated using the Maximum Likelihood Estimation procedure, the Bayesian approach with flat prior distributions and the L-Moments methodology.

From Fig. (6) and Table 2, the L-Moments approach seems to lead to higher return levels of wave height, while the Bayesian methodology with nearly flat priors to higher return levels of storm surge. It is obvious that the Maximum Likelihood Estimation procedure underestimates the return values of both variables, compared to the other two ap- 
(a) MLE and bayesian methods

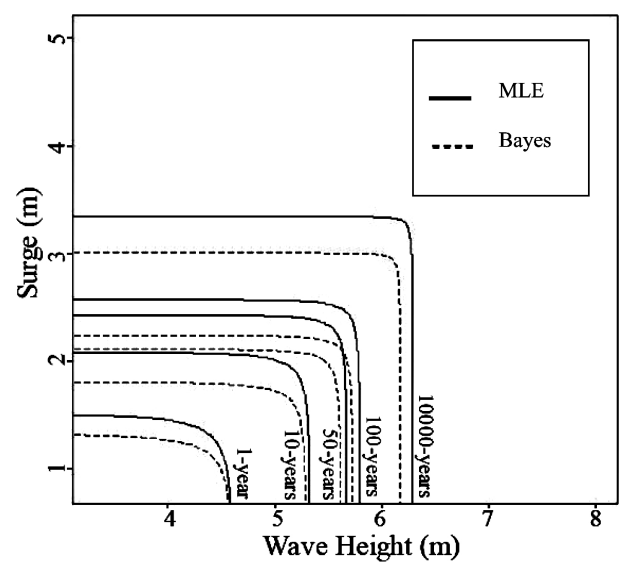

(b) MLE and LM methods

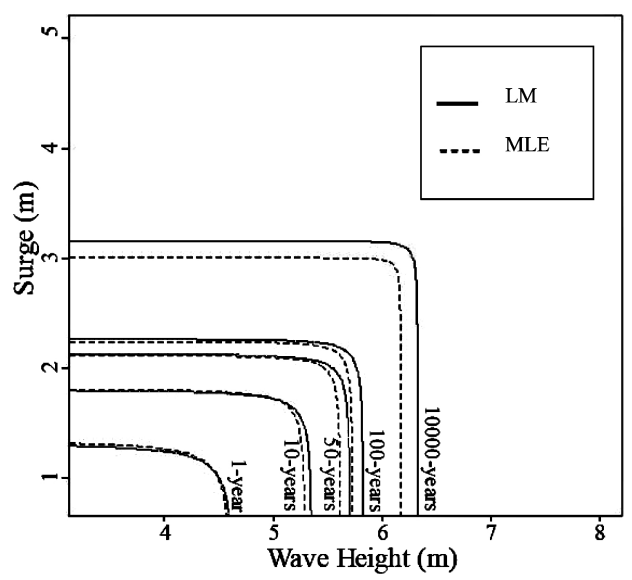

Fig. (6). Bivariate return levels with three methods of estimating the marginal parameters of wave heights and surges (station Swb).

Table 2. Maximum Values of Return Levels for Wave Height and Storm Surge, using three different Methods of estimating the Marginal Distributions of the two Variables (Station Swb)

\begin{tabular}{|c|c|c|c|c|c|c|}
\hline \multirow{2}{*}{ Return Period } & \multicolumn{2}{|c|}{ Maximum Likelihood } & \multicolumn{2}{c|}{ Bayesian Estimation } & \multicolumn{2}{c|}{ L-Moments } \\
\cline { 2 - 7 } & Wave Height (m) & Storm surge (m) & Wave Height (m) & Storm surge (m) & Wave Height (m) & Storm surge (m) \\
\hline \hline 1 year & 4.58 & 1.32 & 4.59 & 1.49 & 4.59 & 1.31 \\
\hline 10 years & 5.26 & 1.79 & 5.31 & 2.07 & 5.34 & 5.70 \\
\hline 50 years & 5.59 & 2.12 & 5.65 & 2.44 & 5.82 & 2.12 \\
\hline 100 years & 5.70 & 2.22 & 5.78 & 2.55 & 3.37 & 3.15 \\
\hline 10000 years & 6.16 & 3.02 & 6.29 & 35 & & 2.33 \\
\hline
\end{tabular}

proaches. When compared to the Bayesian approach, this underestimation reaches $2 \%$ and $11 \%$ for wave height and storm surge, respectively. The larger difference between the two methods for surge return levels can be attributed to large divergences in the fitting of marginal surge distributions, using the two methods of estimation. These divergences are subsequently reflected to the bivariate analysis. When the LMoments approach is used, wave return levels are estimated higher, compared to those of the Maximum Likelihood procedure, up to $2.8 \%$, while differences for surge levels up to 4.3\%. The Bayesian estimation procedure seems to overestimate the joint exceedance probability of extreme events for high values of storm surge (up to $15.5 \%$ for storm surge values), compared to the L-Moments approach.

The comparison of the results coming from the three different methods of estimation reveals the impact of marginal characteristics of the variables and of the different estimation methods on joint exceedance probabilities. The combination of the method of L-Moments (or the Bayesian method) for wave height and of the Bayesian estimation for storm surge can lead to the most conservative results in terms of bivariate return levels.

Using the margins resulting from the three different estimation methods, an approach to estimate the failure area of a particular structure under extreme sea conditions is presented. The characteristics of the structure used in the pre- sent work are those proposed by Coles and Tawn [3] and introduced in Section 3. In Fig. (7), for station Swb of the Dutch coast and design height of the seawall $v_{p}=10 \mathrm{~m}$, the boundary function of equation (14) is presented for design overtopping discharge per unit length $Q\left(v_{p}, z\right)=0.002 \mathrm{~m}^{3} / \mathrm{sm}$. Each contour corresponds to a different wave period in the range of $T=4,6,8,10,12,14 \mathrm{~s}$ from top to bottom.

For all different return periods, a large number of different combinations of wave height and surge with the same joint exceedance probability is estimated. Only one of these combinations is the critical combination in terms of the response of the structure under study, namely in terms of overtopping discharge. For the seawall examined in the present study, the worst case combination of wave height and surge, for a given value of design height of the seawall $v_{p}$, is estimated as the intersection point of the contour line of equal joint exceedance probability of the previously mentioned variables and the contour line corresponding to equal discharge rates $Q\left(v_{p}, z\right)$. Fig. (8) shows the critical combination of wave height and surge for the three different methods of estimation of the marginal distributions of the two variables: a) the Maximum Likelihood Estimation approach, b) the Bayesian procedure and c) the L-Moments approach. The contours of equal joint exceedance probability are defined for station Swb and for a return period of 4000 years, in accordance with the typical standards of the southern part of the Dutch coast on the North Sea. 


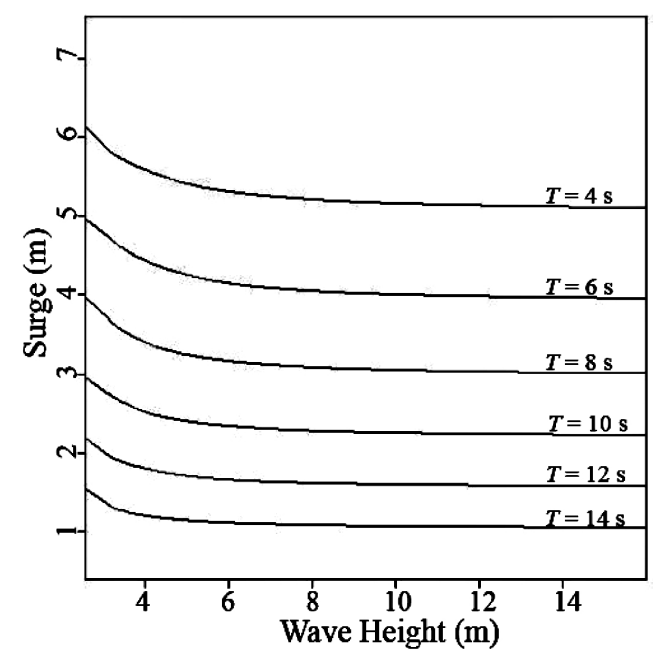

Fig. (7). Contours of design overtopping discharge $Q\left(v_{p}, z\right)=0.002$ $\mathrm{m}^{3} / \mathrm{sm}$ for wave periods $T=4,6,8,10,12,14 \mathrm{~s}$.

In Fig. (8), the intersection points of the contour lines are estimated for wave periods of $T=8.7 \mathrm{~s}$ for the Maximum Likelihood Estimation procedure, $T=7.9 \mathrm{~s}$ for the Bayesian estimation and $T=8.4 \mathrm{~s}$ for the L-Moments approach. The critical combinations of wave height $(x)$ and surge $(y)$, as estimated from Fig. (8), are for the Maximum Likelihood Estimation procedure $x_{4000 \text { years }}=5.76 \mathrm{~m}$ and $y_{4000 \text { years }}=2.88 \mathrm{~m}$ $(T=8.7 \mathrm{~s})$, for the Bayesian estimation approach $x_{4000}$ years $=5.90 \mathrm{~m}$ and $y_{4000}$ years $=3.19 \mathrm{~m}(T=7.9 \mathrm{~s})$ and for the LMoments approach $x_{4000}$ years $=5.92 \mathrm{~m}$ and $y_{4000 \text { years }}=2.97 \mathrm{~m}$ $(T=8.4 \mathrm{~s})$. The Bayesian and L-Moments wave height estimates are almost identical, while regarding the surge, the Bayesian estimates are the highest. With regard to the Maximum Likelihood Estimation procedure, the values of

(a) Maximum Likelihood Estimation

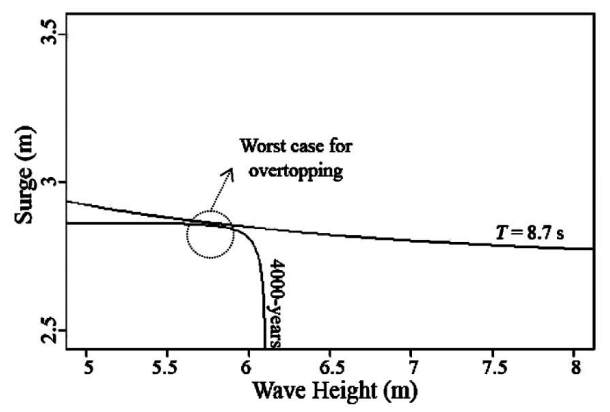

wave height when marginal parameters from the Bayesian estimation approach are used, are estimated higher up to $2.5 \%$, while the respective values of surge up to $10.7 \%$. The wave period, $T$, in the latter case is lower up $9.2 \%$. It can be noticed (Fig. (8)) that the equal overtopping curve lies towards the top left of the diagram for all three different methods of estimation of the marginal parameters of the two variables.

\section{DISCUSSION AND CONCLUSIONS}

In the present work a bivariate process of extreme waves and storm surges at the Dutch station Schouwenbank (Swb) was considered. After the selection of an appropriate bivariate sample to be analyzed using extreme value methodologies, the analysis proceeded with the estimation of joint exceedance probabilities using the simple bivariate logistic model and a sequential estimation procedure, where the parameters of the margins of the bivariate distribution were defined by: a) Maximumum Likelihood Estimation (MLE), b) Bayesian estimation with flat prior distributions and c) the L-Moments estimation procedure. Using the margins resulting from the three different methods of estimation, an approach to estimate the failure region of a particular structure under extreme sea conditions was presented. The main conclusions of the paper can be summarized as follow:

a. The variables of wave height and storm surge are both related to local meteorological conditions. Thus, it is expected that dependence at their extreme levels can be high enough for the variables to be considered consistent with asymptotic dependence. This assumption leads to the use of a bivariate distribution from the family of MVE to model their dependence structure. In the present work, the symmetric bivariate logistic model was proven to be adequate.

(b) Bayesian approach

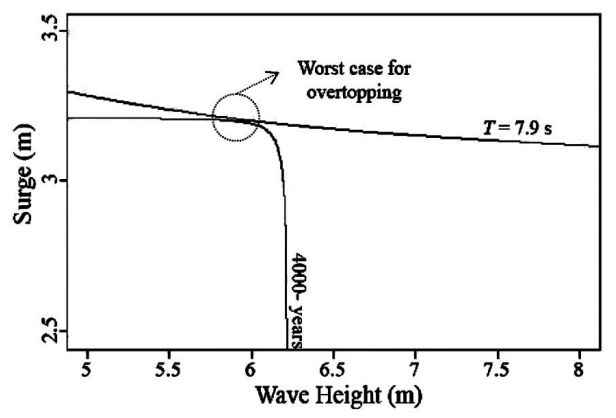

(c) L-Moments Procedure

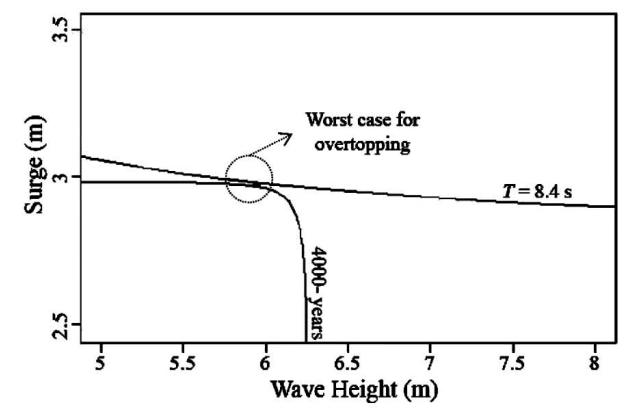

Fig. (8). Estimation of the worst case combination of significant wave height and surge with return period of 4000 years for three different methods of estimation of their marginal distributions (station Swb). 
b. The method of estimation of the marginal characteristics of wave height and storm surge has a large impact on joint exceedance probabilities of the two variables. The Maximum Likelihood Estimation (MLE) approach underestimates the probability of exceedance of extreme joint events of waves and surges compared to those of the Bayesian with flat prior distributions and of the L-Moments procedures. When the Bayesian estimation procedure is used for the marginal distribution of the storm surge, bivariate return levels are more conservative compared to the other two methods of estimation, while for wave height the Bayesian estimation and the L-Moments approach give really close results. It should be noted that in each case the method of estimation of the marginal parameters of the variables involved, which leads to the most conservative results in terms of joint exceedance probabilities, depends critically upon the characteristics of the extreme sample under study.

c. When overtopping of a coastal structure is considered as the main cause of flooding in a certain area, the worst case combination of wave height and storm surge, for a given design value of the height of the structure $v_{p}$, can be estimated as the intersection point of the contour line of joint exceedance probabilities of the variables with the contour line which corresponds to equal overtopping discharge rate per unit length of the structure, $Q\left(v_{p}, z\right)$.

d. The equal overtopping curve of the structure under study lies towards the area of the diagram of the two variables, where surge level is higher for all three different methods of estimation of the marginal parameters of the wave height and the storm surge.

e. Comparing the worst case combinations for the seawall under study, the Bayesian and L-Moments wave height estimates are almost identical, while the Bayesian estimates give the highest surge. Wave height and surge, when marginal parameters from the Bayesian estimation approach are used, are estimated up to $2.5 \%$ and $10.7 \%$ higher than those of the Maximum Likelihood Estimation procedure.

\section{ACKNOWLEDGEMENTS}

The authors want to thank the Dutch National institute RIKZ for providing data of the Dutch coast. The work described in this publication was supported by the European Community's Sixth Framework Programme through the grant to the budget of the Integrated Project FLOODsite, Contract GOCE-CT-2004-505420. The paper reflects the authors' views and not those of the European Community. Neither the European Community nor any member of the FLOODsite Consortium is liable for any use of the information in this paper.

\section{NOTATION - GLOSSARY}

$\begin{array}{lll}\text { MVE } & = & \begin{array}{l}\text { Multivariate Extreme Value distribu- } \\ \text { tions }\end{array} \\ \text { MLE } & = & \begin{array}{l}\text { Maximum Likelihood Estimation pro- } \\ \text { cedure }\end{array} \\ \text { LM } & = & \text { L-Moments approach } \\ \text { GPD } & = & \text { Generalized Pareto Distribution } \\ u & = & \text { Appropriate threshold for variable } X\end{array}$

\begin{tabular}{|c|c|c|}
\hline$n_{u}$ & $=$ & Observations exceeding $u$ \\
\hline$x_{\max }$ & $=$ & Largest value among all $X_{i}$ \\
\hline$X_{1}, X_{2}$ & $=$ & $\begin{array}{l}\text { Representations of wave height and } \\
\text { surge variables }\end{array}$ \\
\hline$R$ & $=$ & $\left(X_{1}+X_{2}\right) / n$ Radial component \\
\hline$W_{1}$ & $=$ & $X_{1} /\left(X_{1}+X_{2}\right)$ angular component \\
\hline$F$ & $=$ & Distribution function of data \\
\hline $\boldsymbol{\theta}$ & $=$ & $\begin{array}{l}(\mu, \sigma, \xi)=\text { Vector of parameters (location, } \\
\text { scale, shape) of distribution fitted to } \\
\text { data }\end{array}$ \\
\hline$L(\boldsymbol{\theta}, x)$ & $=$ & $\begin{array}{l}\text { Likelihood function of data with re- } \\
\text { spect to model parameters }\end{array}$ \\
\hline$f\left(x_{i}, \boldsymbol{\theta}\right)$ & $=$ & Density functions of data \\
\hline$f_{\mu}(\cdot), f_{\varphi}(\cdot), f_{\xi}(\cdot)$ & $=$ & $\begin{array}{l}\text { Normal density functions with mean } \\
\text { zero and variances } v_{\mu}, v_{\varphi}, v_{\xi} \text {, respec- } \\
\text { tively }\end{array}$ \\
\hline$\pi(\theta \mid x)$ & $=$ & Posterior density of parameters vector $\boldsymbol{\theta}$ \\
\hline$\pi(\boldsymbol{\theta})$ & $=$ & Prior density for vector of parameters $\boldsymbol{\theta}$ \\
\hline$L(\boldsymbol{\theta} ; x)$ & $=$ & $\begin{array}{l}\text { A likelihood for } \boldsymbol{\theta} \text { based on an observed } \\
\text { set of exceedances } x\end{array}$ \\
\hline POT & $=$ & Peaks Over Threshold \\
\hline$\tau_{3}$ & $=$ & The normalized L-Moment $\tau_{3}=\lambda_{3} / \lambda_{2}$ \\
\hline$U, V$ & $=$ & $\begin{array}{l}\text { Transformed pair of variables having } \\
\text { Uniform marginal distributions }\end{array}$ \\
\hline$\chi, \bar{\chi}$ & $=$ & $\begin{array}{l}\text { Measures of extremal dependence in- } \\
\text { troduced by Coles et al. }[14]\end{array}$ \\
\hline$T$ & $=$ & $\begin{array}{l}\text { Structure variable defined to estimate } \\
\text { the coefficient of tail dependence, } \eta\end{array}$ \\
\hline$\eta$ & $=$ & Coefficient of tail dependence \\
\hline$s$ & $=$ & $\begin{array}{l}\text { Transformation of variables to unit Fré- } \\
\text { chet margins }\end{array}$ \\
\hline BVE & $=$ & Bivariate Extreme Value distributions \\
\hline$S_{2}$ & $=$ & $\begin{array}{l}\text { Unit simplex }\left\{\left(W_{1}, W_{2}\right): \sum_{\mathrm{j}=1}^{2} W_{\mathrm{j}}=1, W_{\mathrm{j}}>0 \text {, }\right. \\
\mathrm{j}=1,2\}\end{array}$ \\
\hline$\psi_{\alpha}$ & $=$ & Asymmetry parameter \\
\hline$A$ & $=$ & Dependence function of the data \\
\hline NLLH & $=$ & Negative log-likelihood \\
\hline$G\left(x_{1}, x_{2}\right)$ & $=$ & $\begin{array}{l}\text { Bivariate distribution (the symmetric } \\
\text { logistic model) of } X_{1} \text { and } X_{2}\end{array}$ \\
\hline$z_{1}, z_{2}$ & $=$ & $\begin{array}{l}\text { transformed GPD margins of the vari- } \\
\text { ables } X_{1} \text { and } X_{2}\end{array}$ \\
\hline$r$ & $=$ & $\begin{array}{l}\text { Dependence parameter of bivariate lo- } \\
\text { gistic model }\end{array}$ \\
\hline$A_{\mathrm{v}}$ & $=$ & $\begin{array}{l}\left\{\mathbf{x} \in \mathbf{R}^{d}: b(\mathbf{x} ; \mathbf{v})>0\right\} \text { failure region of a } \\
\text { coastal structure }\end{array}$ \\
\hline$b$ & $=$ & $\begin{array}{l}\text { boundary function } \mathbf{R}^{d} \times V \rightarrow \mathbf{R} \text {, where } V \\
\text { is the design parameter space of } \mathbf{v}\end{array}$ \\
\hline$v$ & - & The height of the seawall \\
\hline
\end{tabular}




\begin{tabular}{|c|c|c|}
\hline$X_{1}^{*}, X_{2}^{*}, X_{3}$ & $=$ & $\begin{array}{l}\text { The inshore significant wave height, } \\
\text { wave period and surge, respectively }\end{array}$ \\
\hline$m$ & $=$ & $\begin{array}{l}\text { The mean sea level at the area under } \\
\text { study }\end{array}$ \\
\hline$z$ & $=$ & The mean tidal level \\
\hline$Q(v, z)$ & $=$ & $\begin{array}{l}\text { Expected overtopping discharge rate } \\
\text { per unit length of the seawall }\end{array}$ \\
\hline$\alpha_{1}, \alpha_{2}$ & $=$ & $\begin{array}{l}\text { Dimensionless constants depending on } \\
\text { the form and characteristics of the sea- } \\
\text { wall design }\end{array}$ \\
\hline$Q\left(v_{p}, z\right)$ & $=$ & $\begin{array}{l}\text { Design overtopping discharge per unit } \\
\text { length of the seawall }\end{array}$ \\
\hline$v$ & $=$ & Realisations of variables $X, Y$ \\
\hline
\end{tabular}

\section{REFERENCES}

[1] P. J. Hawkes, B. P. Gouldby, J. A. Tawn, and M. W. Owen, "The joint probability of waves and water levels in coastal engineering design”, J. Hydraulic Res., vol. 40(3), pp. 241-251, 2002.

[2] S. G. Coles, and J. A. Tawn, "Modelling extreme multivariate events", J. R. Stat. Soc. Series B Stat. Methodol., vol. 53(2), pp. 377-392, 1991.

[3] S. G. Coles, and J. A. Tawn, "Statistical methods for multivariate extremes: An application to structural design", Appl. Stat., vol. 43(1), pp. 1-48, 1994.

[4] H. Joe, R. L. Smith, and I. Weissman, "Bivariate threshold methods for extremes", J. R. Stat. Soc. Series B., vol. 54, pp. 171-183, 1992.

[5] S. Zachary, G. Feld, G. Ward, and J. Wolfram, "Multivariate extrapolation in the offshore environment", Appl. Ocean Res., vol. 20, pp. 273-295, 1998.

[6] DEFRA/ Environment Agency Flood and Coastal Defence R\&D Programme, Best Practice in Coastal Flooding, R\&D Technical Report FD2206/TR1, Workshop Version issued 24/09/03, HR Wallingford Report TR 132, 2003.

[7] A. Butler, "Statistical Modelling of Synthetic Oceanographic Extremes", Ph.D. thesis, Lancaster University, London, UK, 2005.

[8] C. W. Li, and Y. Song "Joint probability analysis of extreme waves and water levels at a typhoon invaded coastal region", In: Proceedings of XXXI IAHR Congress, 2005, pp. 3924-3934.

[9] S. P. Yeh, and S. P. Ou, "Joint probability analysis of waves and water level during typhoons", In: Proceedings of the Third Chinese-German Joint Symposium on Coastal and Ocean Engineering, 2006, [Online] Available:

http://www.comc.ncku.edu.tw/joint/joint2006/pdf/68\%20CHM\%20 06.pdf [Accessed $28^{\text {th }}$ October 2009]

[10] T. A. Buishand, "Bivariate extreme-value data and the station-year method", J. Hydrol., vol. 69, pp. 77-95, 1984.

[11] T. A. Buishand, "Extreme rainfall estimation by combining data from several sites", Hydrol. Sci. J., vol. 36, pp. 345-365, 1991.

[12] M. Y. Dales, and D. W. Reed, Regional Flood and Storm Hazard Assessment, Institute of Hydrology no 102, Institute of Hydrology, Wallingford, Oxfordshire, OX10 8bb, UK, 1989.

[13] J. P. Chilès, and P. Delfiner, Geostatistics: Modelling Spatial Uncertainty, Wiley, New York, 1999.
[14] S. Coles, J. Heffernan, and J. Tawn, "Dependence measures of extreme value analysis", Extremes, vol. 2(4), pp. 339-365, 1999.

[15] S. Coles, An Introduction To Statistical Modelling of Extreme Values, Springer Series in Statistics, Springer, Berlin, 2001.

[16] A. Ledford, and J. A. Tawn, "Diagnostics for dependence within time series extremes", J. R. Stat. Soc. Series B, vol. 65(2), pp. 521543, 2003.

[17] C. Svensson, and D. A. Jones, "Dependence between sea surge, river flow and precipitation in South and West Britain", Hydrol. Earth Syst. Sci., vol. 8(5), pp. 973-992, 2004.

[18] P. Galiatsatou, and P. Prinos, "Analysis of dependence in a bivariate process of extreme waves and surges", In: Proceedings of the $I^{\text {st }}$ International Conference on Coastal Zone Management and Engineering in the Middle East, Dubai 2005, pp. 221-225

[19] P. Galiatsatou, "Joint exceedance probabilities of extreme waves and storm surges", In: Proceedings of XXXIII Congress of IAHR, 2007, pp. 780 (abstract) - JFK Competition, (The full paper in the electronic proceedings of the conference)

[20] P. Galiatsatou, and Prinos, P., "Analysis of extreme rainfall events using a Poisson process", In: Proceedings of the $10^{\text {th }}$ National Conference E.Y.E, Xanthi 2006, Greece, pp. 47-54.

[21] P. Galiatsatou, P. Prinos, and A. Sánchez-Arcilla, "Estimation of extremes. Conventional versus Bayesian techniques", J. Hydraulic Res., vol. 46(2), pp. 211-223, 2008.

[22] A. Sánchez-Arcilla, J. Gomez-Aguar, J. J. Egozcue, M. I. Ortego, P. Galiatsatou, and P. Prinos, "Extremes from scarce data. The role of Bayesian and scaling techniques in reducing uncertainty", J. Hydraulic Res., vol. 46(2), pp. 224-234, 2008.

[23] R. L. Smith, "Extreme value analysis of environmental time series: an example based on ozone data (with discussion)", Stat. Sci., vol. 4, pp. 367-393, 1989.

[24] P. Galiatsatou, and P. Prinos, "Statistical models for bivariate extremal analysis of a spatial process", J. Hydraulic Res., vol. 46(2), pp. $257-270,2008$.

[25] A. Repko, P. H. A. J. M. van Gelder, H. G. Voortman, and J. K. Vrijling, "Bivariate description of offshore wave conditions with physics- based extreme value statistics", Appl. Ocean Res., vol. 26, pp. 162-170, 2004.

[26] P. H. A. J. M. van Gelder, "Risk-based Design of Civil Structures", Ph.D. thesis, University of Technology, Delft, The Netherlands, 1999.

[27] A. Stephenson, and J. Tawn, "Bayesian inference for extremes: Accounting for the three extremal types", Extremes, vol. 7(4), pp. 291-307, 2004

[28] J. R. M. Hosking, "L-Moments: Analysis and estimation of distributions using linear combinations of order statistics", J. R. Stat. Soc. Series B, vol. 52, pp. 105-124, 1990.

[29] M. D. Pandey, P. H. A. J. M. van Gelder, and J. K. Vrijling, "The estimation of extreme quantiles of wind velocity using L-moments in the peaks-over-threshold approach", J. Structural Safety, vol. 23(2), pp. 179-192, 2001.

[30] J. A. Tawn, "Bivariate extreme value theory: Models and estimation", Biometrika, vol. 75, pp. 397-415, 1988.

[31] DEFRA/ Environment Agency Flood and Coastal Defence R\&D Programme, Joint Probability: Dependence Mapping and Best Practice, Technical report on dependence mapping, R\&D Technical Report FD2308/TR1, 2005.

[32] M. J. Tucker, A. P. Carr, and E. G. Pitt, "The effect of an offshore bank in attenuating waves", Coastal Eng., vol. 7, pp. 113-144, 1983.

(C) Galiatsatou and Prinos; Licensee Bentham Open.

This is an open access article licensed under the terms of the Creative Commons Attribution Non-Commercial License (http://creativecommons.org/licenses/by-nc/3.0/) which permits unrestricted, non-commercial use, distribution and reproduction in any medium, provided the work is properly cited. 